\title{
EFEITOS DA CRIOTERAPIA VS CORRENTES DIADINÂMICAS NA REDUÇÃO DO EDEMA DE PATA EM RATAS
}

\author{
Juliano Yasuo Oda ${ }^{1}$ \\ Fabrícia Lorca dos Santos Garnés ${ }^{2}$ \\ Anni Jaquelini Martins ${ }^{2}$ \\ Orlando Seiko Takemura ${ }^{3}$
}

ODA, J. Y.; GARNÉS, F. L. dos S.; MARTINS, A. J.; TAKEMURA, O. S. Efeitos da crioterapia vs correntes diadinâmicas na redução do edema de pata em ratas. Arq. Ciênc. Saúde UNIPAR, Umuarama, v. 18, n. 2, p. 107-111, maio/ago. 2014.

\begin{abstract}
RESUMO: O edema inflamatório agudo é considerado um problema para a reabilitação, pois acarreta perda da mobilidade e dor, comprometendo a funcionalidade do indivíduo, sendo importante sua diminuição pós-lesão. O presente estudo comparou os efeitos da crioterapia e das correntes diadinâmicas de Bernard (CDB) na redução de edema induzido experimentalmente. O experimento incluiu 15 ratas Wistar com 256,16 $\pm 15,48$ g, divididas em três grupos constituídos de 5 animais cada, sendo G1, G2 e G3. Foi realizado a hidropletismografia da pata direita de cada animal, seguida da indução do edema em todos os animais com carragenina $1 \%$ e solução salina $0,9 \%$. Os animais foram reavaliados após 60; 120 e 180 minutos da indução do edema e após o tratamento. Os animais do G1 foram tratados através da crioterapia via imersão por 10 minutos; G2, submetidos à aplicação das CDB utilizando as correntes difásica, monofásica e curtos períodos, por 9 minutos e G3 representou o grupo controle. Para G1, o volume deslocado inicialmente foi 1,68 $\pm 0,18 \mathrm{ml}$; após 180 minutos 2,8 \pm 0,28 ; e após o tratamento com crioterapia $2,4 \pm 0,28 \mathrm{ml}$. Para G2, os valores foram $1,76 \pm 0,22 ; 2,72 \pm 0,33$; e posteriormente à aplicação das correntes, o valor foi $2,32 \pm 0,33 \mathrm{ml}$. Para G3, os valores foram 1,6 $\pm 0 ; 3,04 \pm 0,35$; ao término do tratamento proposto para G1 e G2, o G3 apresentou 3,12 $\pm 0,33 \mathrm{ml}$. Os resultados demonstraram redução não significativa do edema comparando G1 e G2, porém apresentou diminuição significativa $(\mathrm{p}<0,01)$, quando comparado G1 e G2 com o G3. As duas modalidades terapêuticas avaliadas foram eficazes na redução do edema induzido experimentalmente, no entanto não diferiram significativamente quando comparadas entre si.

PALAVRAS-CHAVE: Inflamação; Tratamento; Termoterapia (crioterapia); Eletroterapia (correntes diadinâmicas de Bernard).
\end{abstract}

\section{EFFECTS OF CRYOTHERAPY VS DIADYNAMIC CURRENTS ON THE REDUCTION OF THE RATS PAW EDEMA}

\begin{abstract}
Acute inflammatory edema is considered a problem for the rehabilitation; because it leads to mobility loss and pain endangering the individual functionality, being important its reduction at post-lesion phase. The present study compared the effects of cryotherapy and Bernard's Diadynamic Currents (BDC) on reduction of a pharmacologically induced edema. The trial included 15 Wistar female rats with $256.16 \pm 15.48 \mathrm{~g}$, divided into three groups: G1 under cryotherapy treatment through immersion for 10 minutes; G2 under BDC application using diphasic current, monophasic current and short periods for a nine minutes, and G3 as control group. The volume measurement of right paw of each animal followed by edema induction in all animals with $1 \%$ carragenin solution was carried out by hidropletismography. The animals were re-evaluated 60;120; and 180 minutes after the edema induction and treatment. For animals of G1 group, initial dislocated volume was $1.68 \pm 0.18 \mathrm{ml}$; after 180 minutes, $2.8 \pm 0.28$; and after cryotherapy treatment, $2.4 \pm 0.28 \mathrm{ml}$. For G2, values were $1.76 \pm 0.22 ; 2.72 \pm 0.33$; and after currents application, $2.32 \pm 0.33 \mathrm{ml}$. For G3, values were $1.6 \pm 0 ; 3.04 \pm 0.35$; at the end of treatment proposed to G1 and G2, G3 showed $3.12 \pm 0.33 \mathrm{ml}$. The results showed non-significant reduction when comparing G1 and G2 groups; however, significant reduction ( $\mathrm{p}<0,01$ ), was observed when comparing G1 and G2 with G3 group. Both therapeutic modalities were efficient in reducing the pharmacologically induced edema, although they did not significantly differ when compared each other.

KEYWORDS: Inflammation; Treatment; Thermotherapy (cryotherapy); Electrotherapy (Bernard's diadynamic currents).
\end{abstract}

\section{Introdução}

A inflamação pode ser considerada uma resposta estereotipada, com caráter defensivo, que tem como finalidade eliminar a causa e proteger a integridade dos tecidos, a qual ocorre sob os efeitos dos estímulos nocivos, sendo uma de suas características a presença de exsudato (SOUZA, et al., 2004). Kumar et al. (2010), relatam que a marca da inflamação aguda é o aumento da permeabilidade vascular, o que provoca o extravasamento de um líquido rico em proteína (exsudato) para o interstício. A perda de proteínas do plasma reduz a pressão osmótica intravascular e eleva a pressão osmótica do líquido intersticial. Com a pressão hidrostática elevada devido a vasodilatação, ocorre um fluxo acentuado de líquido que se acumula no tecido intersticial denominado de edema.

O edema é considerado um problema para a rea- bilitação, pois a sua formação pode comprimir terminações nervosas e induzir o ciclo dor-espasmo-dor, que reduz a força muscular e amplitude de movimento, comprometendo a funcionalidade da estrutura, sendo importante a sua diminuição imediata após a lesão. Portanto, é necessária uma intervenção terapêutica que tenha por finalidade a eliminação, o controle ou a redução do edema (SOUZA, et al., 2004; KAVAT, et al., 2013).

Diante disso, dentre algumas formas de tratamentos para a diminuição do edema e de seus efeitos deletérios, estão a crioterapia e as correntes diadinâmicas de Bernard (CDB). De acordo com Waterman et al. (2013), diversos estudos clínicos apóiam a evidência empírica para o uso de gelo na redução do edema. Segundo Lianza (2007), a utilização da termoterapia por subtração é comumente utilizada nas afecções músculo-esqueléticas, principalmente na fase aguda dos processos traumáticos e inflamatórios, por auxiliar na re-

DOI: https://doi.org/10.25110/arqsaude.v18i2.2014.5175

${ }^{1}$ Fisioterapeuta, Mestre em Ciências Morfofuncionais, Professor de Anatomia Humana - UFMS - Universidade Federal de Mato Grosso do Sul. Email: juliano.yasuo@ufms.br

${ }^{2}$ Fisioterapeuta graduada pela UNIPAR - Universidade Paranaense.

${ }^{3}$ Farmacêutico Doutor em Bioquímica, professor de Fitoterapia - UNIPAR - Universidade Paranaense. 
dução do processo inflamatório e na formação de edema. A redução do edema que acompanha a aplicação da crioterapia após uma lesão aguda pode ser atribuída a vasoconstrição imediata das arteríolas e vênulas, o que reduz a circulação local e, portanto, reduz o extravasamento de líquidos para o espaço intersticial (WATERMAN et al., 2013).

Guirro e Guirro (2004) descrevem que o frio não produz apenas alterações vasculares, mas também metabólicas. Uma vez que a redução da temperatura local diminui o metabolismo, a demanda de oxigênio e a nutrição. $\mathrm{O}$ menor consumo de oxigênio possibilita uma sobrevida por um maior período de isquemia ou de diminuição parcial de circulação, evitando assim a hipóxia secundária e, conseqüentemente, a morte celular. Isso leva a uma menor extensão do tecido lesado, o que diminui as proteínas livres e a pressão oncótica do tecido, reduzindo então o edema.

Outra modalidade terapêutica descrita na literatura como uma possível forma para a redução do edema são as correntes diadinâmicas de Bernard. São correntes alternadas senoidais de baixa freqüência (50 a $100 \mathrm{~Hz}$ ) com retificação monofásica ou difásica (REINERT, et al., 2005). A combinação entre as correntes monofásica e difásica geram as correntes de curtos e longos períodos (AMATUZZI, CARAZZATO e GREVE, 2004). Rodrigues e Guimarães (1998) apontam para a existência de mais uma corrente, a ritmo sincopado.

A corrente difásica (DF) provoca analgesia e relaxamento muscular, porém é de curta duração e rotineiramente é utilizada como preparatório para a aplicação das demais correntes diadinâmicas. A corrente de curtos períodos (CP) produz forte efeito analgésico e vasomotor, sendo indicada para a redução de edemas e dor, pois aumenta consideravelmente o fluxo sanguíneo, produzindo contrações musculares rítmicas que contribuem para a diminuição do edema, dor e espasmos musculares (AMATUZZI, CARAZZATO e GREVE, 2004). A corrente monofásica (MF) é recomendada para estimulação inespecífica do tecido conjuntivo, pois acelera o seu metabolismo (GUIRRO e GUIRRO, 2004).

De acordo com Rodrigues e Guimarães (1998), as CDB promovem o efeito de "fuga" de líquidos do pólo positivo para o pólo negativo, fenômeno este conhecido como endosmose. Além disso, outros efeitos promovidos por estas correntes justificam sua ação sobre o edema como: aumento do fluxo linfático, aumento da drenagem venosa e da circulação sangüínea local.

Entre os efeitos fisiológicos da crioterapia e das $\mathrm{CDB}$, se destaca a diminuição do edema. No entanto, são escassos os estudos que realizem a comparação entre essas duas modalidades terapêuticas na redução do edema, tal fato, foi relevante para a elaboração do presente estudo, que poderá contribuir com a literatura científica a cerca desse assunto.

O presente estudo teve por objetivo comparar os efeitos da crioterapia e das CDB na redução do edema de pata de rata induzido pela aplicação de carragenina a $1 \%$.

\section{Material e Método}

O experimento foi realizado no laboratório de Farmacologia da Universidade Paranaense - UNIPAR, após a aprovação no Comitê de Ética em Pesquisa envolvendo Experimentação Animal, de acordo com o protocolo 04/2006.

\section{Animais}

Foram utilizados 15 animais de laboratório, Rattus norvegicus da linhagem Wistar, com idade de 04 meses, fêmeas e com peso de 256,16 $\pm 15,48$ g, provenientes do Biotério da Universidade Paranaense - Umuarama, Pr.

Os animais foram mantidos numa condição padrão de temperatura a $23 \pm 2^{\circ} \mathrm{C}$, com ciclo claro-escuro de $12 \mathrm{~h}$, sendo alimentadas com água e ração peletizada tipo Purina ad libitum. Cada grupo experimental foi constituído de 05 animais, sendo o G1 - animais que foram tratados pela crioterapia; G2 - submetidos ao tratamento através das CDB; e G3 - que representaram o grupo controle, e que não receberam nenhuma forma de tratamento.

\section{Procedimentos Experimentais}

Inicialmente foi realizada a tricotomia do membro posterior direito dos animais de todos os grupos. Em seguida, realizou-se uma marcação de $4 \mathrm{~cm}$ no membro posterior direito tendo como referência o $4^{\circ}$ dedo. Os animais de todos os grupos foram pesados, em seguida foram sedados utilizando uma câmara saturada com éter etílico. Através do uso de um pletismógrafo adaptado foi mensurado o volume da pata direita de cada animal através da média de três valores determinados sempre pelo mesmo observador. Para padronizar a avaliação foi utilizada a marcação realizada no membro posterior direito.

Após a mensuração, foi induzido o edema na pata direita dos animais de todos os grupos, através de uma injeção subplantar, contendo $0,1 \mathrm{ml}$ de uma associação de carragenina a $1 \%$ e solução salina a $0,9 \%$ de acordo com Winter et al. (1962).

Para assegurar que todos os animais fossem tratados após 180 minutos da indução do edema, foi determinado um intervalo de aplicação do agente flogístico, sendo que para os animais do G1 foi utilizado um intervalo de 10 minutos entre as aplicações; para o G2, intervalo de 15 minutos; e para G3, 5 minutos.

O volume da pata direita de todos os grupos foi reavaliado através da hidropletismografia nos tempos 60, 120 e 180 minutos após a administração do agente flogístico. Os resultados foram expressos como aumento do volume em mililitros (ml).

Após 180 minutos da indução do edema, todos os animais foram anestesiados através de uma injeção intraperitoneal contendo uma associação de Ketamina $60 \mathrm{mg} / \mathrm{kg}$ e Xilazina $8 \mathrm{mg} / \mathrm{kg}$, de acordo com o CETEA - Comitê de Ética em Experimentação Animal - UFMG. Posteriormente os animais foram tratados de acordo com o protocolo proposto.

O grupo G1 foi submetido a apenas uma aplicação de crioterapia por imersão durante 10 minutos, na qual a região edemaciada foi posicionada em um recipiente contendo água e gelo, a uma temperatura de $5^{\circ} \mathrm{C}$, de acordo com técnica utilizada por Souza et al. (2004).

O grupo G2 foi submetido a apenas uma aplicação das CDB, com a utilização do aparelho ENDOPHASYS D ELF0204 da marca KLD - Biosistemas Equipamentos Eletrônicos Ltda, verificado quanto ás variações de calibração e o ambiente no qual foi realizado o experimento era isento de ondas eletromagnéticas. Utilizou - se a técnica vasotró- 
pica, posicionando o eletrodo do pólo negativo na região da coxa direita e o eletrodo do pólo positivo foi fixado em um recipiente de alumínio contendo água. Foram aplicadas as correntes DF, MF e CP, respectivamente, por 3 minutos cada, perfazendo um total de 9 minutos, de acordo com Reinert et al. (2005).

Para a aplicação das correntes foram adaptados eletrodos de 1,5 cm X 1,5 cm de acordo com a área a ser tratada. E seguindo o protocolo de Chantraine et al. (1998); Pérez et al. (2004) utilizou-se uma intensidade de $0,3 \mathrm{~mA}$. O grupo G3 constou de animais com lesão induzida farmacologicamente na pata direita, os quais não foram submetidos a nenhuma forma de tratamento.

Após a execução dos procedimentos terapêuticos, o volume da região lesada foi reavaliado através da hidropletismografia, tanto nos animais pertencentes aos grupos G1 e G2 como nos animais do grupo controle, para acompanhamento do edema diante da crioterapia e da aplicação das CDB. Entretanto, é relevante salientar que não foi observada a reação do edema por um longo período de tempo após o tratamento, uma vez que, ao término da última avaliação os animais foram submetidos à eutanásia através de uma dose letal de pentobarbital sódico $150 \mathrm{mg} / \mathrm{kg}$, de acordo com o protocolo de Mervaala et al. (2000).

Os resultados das análises de cada animal foram submetidos ao teste Anova e Tukey`s com nível de significância de $(\mathrm{p}<0,01)$, a fim de verificar a existência de diferença estatisticamente significativa entre os dados analisados.

\section{Resultados}

Através da análise dos dados referentes aos animais do grupo G1 (crioterapia), os valores relacionados a hidropletismografia mostram que o volume deslocado inicialmente pela pata direita foi de $1,68 \pm 0,18 \mathrm{ml}$; após sessenta minutos da indução do edema, foi de 2,08 $\pm 0,18 \mathrm{ml}(23,8 \%)$; depois de cento e vinte minutos, $2,48 \pm 0,18 \mathrm{ml}(47,62 \%)$; após cento e oitenta minutos, $2,8 \pm 0,28 \mathrm{ml}(66,67 \%)$. Em seguida ao tratamento com crioterapia, o volume apresentado foi $2,4 \pm$ $0,28 \mathrm{ml}$ (42,86\%), demonstrando uma diminuição do edema induzido, quando comparado ao grupo controle (Figura 1).

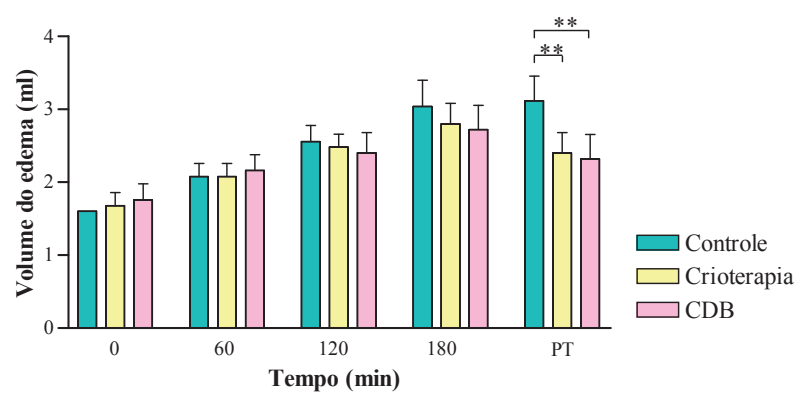

Figura 1: Variação dos valores em mililitros obtidos nos três grupos.

Legenda: CDB. - correntes diadinâmicas de Bernard; PT - Pós tratamento; $(* *)-\mathrm{p}<0,01$.

Para o G2 (CDB), a média inicial foi $1,76 \pm 0,22$ $\mathrm{ml}$ de deslocamento de água. Sessenta minutos após a lesão, passou a 2,16 $\pm 0,22 \mathrm{ml}(22,73 \%)$; depois de cento e vinte minutos, $2,4 \pm 0,28 \mathrm{ml}(36,36 \%)$; após cento e oitenta minu- tos, 2,72 $\pm 0,33 \mathrm{ml}(54,55 \%)$. Posteriormente à aplicação das correntes diadinâmicas o valor apresentado foi $2,32 \pm 0,33$ $\mathrm{ml}(31,82 \%)$, resultado que representou $26 \%$ de diminuição do edema em relação aos animais do grupo controle.

No grupo controle a média de deslocamento de água prévio à lesão era de $1,6 \mathrm{ml}$, alterou-se para 2,08 $\pm 0,18 \mathrm{ml}$ $(30 \%)$ após sessenta minutos; cento e vinte minutos depois da lesão apresentou 2,56 $\pm 0,22 \mathrm{ml}(60 \%)$; após cento e oitenta minutos observou-se 3,04 $\pm 0,35 \mathrm{ml}(90 \%)$. Ao término do tratamento proposto para os grupos G1 e G2, o grupo G3 apresentou um aumento do edema, com um deslocamento de $3,12 \pm 0,33 \mathrm{ml}(95 \%)$ em relação ao valor inicial, resultado demonstrado pela hidropletismografia, o mesmo não acontecendo nos grupos G1 e G2.

Em relação aos dados obtidos no G1 e G2, observou-se que não houve diferença significativa $(p>0,05)$ na redução do edema após a aplicação das modalidades terapêuticas. Apesar da inexistência de diferença estatística, observou-se uma tendência mais acentuada de diminuição do edema em G2 (26\%) quando comparado com G1 (23\%), em relação ao grupo de controle. Comparando os valores dos grupos G1 e G2 com os do grupo G3, a redução do edema foi estatisticamente significativa $(\mathrm{p}<0,01)$.

\section{Discussão}

Após as análises dos resultados apresentados na Figura 1, constatou-se que ambas as técnicas, crioterapia e CDB, resultaram em diminuição do edema induzido experimentalmente nos membros posteriores de ratos. $\mathrm{O}$ uso da crioterapia nas situações de lesões agudas, bem como na reabilitação pós-trauma agudo vem sendo estabelecido devido aos vários benefícios que provoca (GUIRRO, MÁXIMO, 1999; KNIGHT, 2000; HAUSSWIRTH, et al., 2011).

De acordo com Monteiro et al (1997), a aplicação de gelo associado à elevação reduziu o edema induzido por dextrana na pata posterior de ratos. Eles concluíram que a elevação provavelmente diminuiu a pressão hidrostática capilar e aumentou a drenagem linfática; ao passo que a crioterapia produziu vasoconstrição, reduziu o metabolismo, bem como a liberação e ação da histamina nas junções endoteliais. Segundo o estudo de Guirro e Máximo (1997) e Costello et al. (2014), a vasoconstrição decorrente do estímulo das fibras simpáticas e a diminuição da pressão oncótica, juntamente com a diminuição da permeabilidade da membrana, levam a uma redução do edema. No presente estudo pôde-se observar uma redução significativa do edema no grupo G1 em relação ao grupo controle, tal resultado esteve ligado aos efeitos relacionados à crioterapia, também verificados nos estudos citados acima.

Brancaccio et al. (2005), em seu estudo compararam os efeitos da crioterapia na lesão muscular em ratos treinados e sedentários, verificaram a diminuição da presença de edema sendo essa ocorrência maior em animais treinados. Souza et al. (2004), investigaram os efeitos da crioterapia e laser $904 \mathrm{~nm}$ na redução do edema traumático em patas de ratas. Em seu estudo, foi induzido um edema traumático em patas de ratas. Os animais foram divididos em três grupos: um grupo que foi submetido ao tratamento com crioterapia; um grupo que recebeu aplicação de laser $904 \mathrm{~nm}$, e outro grupo que representou o controle. Nos resultados encontra- 
dos, pode-se observar que a crioterapia promoveu maior redução do edema traumático na pata de ratas, quando comparado com o laser $904 \mathrm{~nm}$, apesar de a redução obtida por este ter-se prolongado por mais tempo.

No presente estudo observou-se uma redução do edema através da crioterapia via imersão, sendo que a redução do edema, apesar de não significativa em relação ao grupo G2 (CDB), demonstrou uma diferença significativa em relação ao grupo controle $(\mathrm{p}<0,01)$.

Além dos efeitos da crioterapia, neste estudo foi observada a influência das CDB na redução do edema. Para Rodrigues e Guimarães (1998) as CDB, como correntes polarizadas, podem ser uma alternativa para redução de edema, pela característica de provocar a endosmose. Machado, 2002 apud Carvalho et al. (2005), complementa que a endosmose consiste em um efeito resultante da passagem de corrente contínua pelo tecido, o que provoca a transferência do líquido do pólo positivo para o pólo negativo. Low e Reed (2001) relatam que outros efeitos promovidos pelas CDB que contribuem para a diminuição da inflamação e do edema são: o aumento da ação de bombeamento muscular e da circulação local, e a ocorrência de alterações de permeabilidade da membrana celular.

Carvalho et al. (2005), observaram a atuação das CDB e iontoforese na dor lombar, verificando outra vantagem das CDB que também pode ter contribuído para a melhora do quadro doloroso - a redução do edema pelo mecanismo da endosmose.

Reinert et al. (2005), demonstraram o efeito da eletrosmose apresentada pelas CDB. Nesse estudo, foi induzido edema traumático decorrente de lesões seriadas em patas de ratas. Essas foram divididas em três grupos: um grupo controle; um grupo que recebeu terapia com CDB com o pólo positivo sobre a lesão e o pólo negativo distalmente; e um outro grupo que recebeu terapia com $\mathrm{CDB}$ com pólo negativo sobre a lesão e o pólo positivo distalmente. Observou-se que o grupo controle e aqueles que receberam o pólo negativo sobre a lesão tiveram aumento significante na magnitude do edema. Quando o pólo positivo foi colocado sobre a lesão houve uma tendência à redução do edema, embora não de forma significativa.

No presente estudo observou-se uma redução do edema através da aplicação das correntes diadinâmicas no grupo G2, pôde-se verificar que essa redução foi devido ao efeito da endosmose decorrente do posicionamento do pólo positivo sobre o edema, confirmando os resultados do estudo citado acima.

Os resultados obtidos neste estudo referente às diadinâmicas demonstraram redução significativa do edema em relação ao grupo controle, o qual não foi submetido a nenhuma forma de tratamento, bem como redução não significativa quando comparado com o grupo G1 (crioterapia).

\section{Conclusão}

A análise comparativa realizada no presente estudo, revelou que ambas as modalidades testadas foram eficazes na redução do edema de pata de rata induzido pela aplicação de carragenina a 1\%, quando comparadas com o grupo controle. Entretanto, quando comparadas às duas modalidades terapêuticas, os resultados não demonstraram diferenças significativas.

\section{Referências}

AMATUZZI, M. M.; CARAZZATO, J. G.; GREVE, J. M. A.; Reabilitação em medicina do esporte. 1. ed. São Paulo: Roca; 2004. 317p.

BRANCACCIO, N. et al., Análise de lesão muscular em ratos treinados e sedentários submetidos à crioterapia.

Revista Fisioterapia em Movimento, 18 (1): 59-65; 2005.

CARVALHO, A. R. et al. Correntes diadinâmicas de Bernard e iontoforese no tratamento da dor lombar. Revista Fisioterapia em Movimento, 18 (4): 11-19; 2005.

CHANTRAINE, A.; GOBELET, C.; ZILTENER, J. L. Electrologie et applications. - Encycl. Méd. Chir. (Elsevier, Paris-France), Kinesiothérapie-Médicine physiqueRéadaptation, 26-145-A- 10, 1998, 22p.

COSTELLO, J. et al. Effects of whole body cryotherapy and cold water immersion on knee skin temperature. International Journal of Sports Medicine, 35 (1), p. 35 40, 2014.

\section{GUIRRO, E.; GUIRRO, R. Fisioterapia dermato-}

funcional. 3. ed. São Paulo: Manole; 2004. 120-137p.

GUIRRO, R.; ABIB, C.; MÁXIMO, C. Os efeitos fisiológicos da crioterapia: Uma revisão. Revista Fisioterapia Universidade São Paulo, 6 (2): 164-170; 1999.

HAUSSWIRTH, C. et al. Effects of Whole-Body Cryotherapy vs. Far-Infrared vs. Passive Modalities on Recovery from Exercise-Induced Muscle Damage in Highly-Trained Runners. Plos One 6 (12), 2011.

KAVAT, J. et al. Efeitos das correntes diadinâmicas de Bernard sobre a nociceptividade e edema no trauma tendíneo de ratos. Fisioterapia em Movimento, 26 (3): 617-622, 2013.

KITCHEN, S.; BAZIN, S. Eletroterapia de Clayton. 1. ed. São Paulo: Manole; 1998. 131p.

\section{KNIGHT, K. L. Crioterapia no tratamento das lesões} esportivas. 1. ed. São Paulo: Manole; 2000. 94, 95-204p.

KUMAR, V. et al. Robbins e Cotran - Patologia - Bases patológicas das doenças. 10. ed. São Paulo: Elsevier; 2010.

LIANZA, S. Medicina de reabilitação. 4. ed. Rio de Janeiro: Guanabara; 2007. 496p.

LOW, J.; REED, A. Eletroterapia explicada: Princípios e prática. 3. ed. São Paulo: Manole; 2001. 185-221p.

MERVAALA, E. et al. Blood Pressure-Independent Effects in Rats With Human Renin and Angiotensinogen Genes. 
Hypertension. 2000; 35:587.

MONTEIRO, P. V. et al. Effect of local cryutherapy and elevation on dextran - induced edema in the hind paw of rats. Braz. J. Morphol. Sri. 1997. 14: 265-69p.

PÉREZ, J. G.; FERNÁNDEZ, P. G.; GONZÁLEZ, E. M. Iontoforesis, doses y tratamientos. Rev. La Facultad de Ciencias de la Salud. vol. 2, 3-13, 2004.

REINERT, T. C. et al. Uso de correntes diadinâmicas de Bernard em edema agudo traumático em patas de ratas. Revista Reabilitar, ano 7, n. 26, p. 24-28, $1^{\circ}$ trimestre 2005.

RODRIGUES, E.; GUIMARÃES, C. Manual de recursos fisioterapêuticos. Rio de Janeiro: Revinter; 1998. 43-52p.

SOUZA, D. E. et al. Efeitos da crioterapia e laser $904 \mathrm{~nm}$ na redução de edema traumático em patas de ratas. Revista Reabilitar, 2004; 24 (6): 4-9.

WATERMAN, B. et al. The efficacy of combined cryotherapy and compression compared with cryotherapy alone following anterior cruciate ligament reconstruction. The Journal of knee surgery 25 (2) 155-160, 2012.

WINTER, C. A.; RISLEY, E. A.; NUSS, G. W. Carragenininduced oedema in hind a paw of the rats as an assay for antinflammatory drug. Proc. Soc. Exp. Biolog., v. 3, p. $544-547,1962$. 